\title{
Uniformly normal structure and uniformly generalized Lipschitzian semigroups
}

Ahmed H. Soliman ${ }^{a, *}$, Mohamed A. Barakat ${ }^{a}$

${ }^{a}$ Department of Mathematics, Faculty of Science, Al-Azhar University, Assiut 71524, Egypt.

Dedicated to George A Anastassiou on the occasion of his sixtieth birthday

Communicated by Professor M. Imdad

\begin{abstract}
In this work, we introduce some condition on one-parameter semigroup of self-mappings it is called kuniformly generalized Lipschitzian. The condition is weaker than Lipschitzian type conditions. Also, we show that a k-generalized Lipschitzian semigroup of nonlinear self-mappings of a nonempty closed convex subset $C$ of real Banach space $X$ admits a common fixed point if the semigroup has a bounded orbit and if $k>0$. Our results extending the results due to L.C. Ceng, H. K. Xu and J.C. Yao [5]
\end{abstract}

Keywords: Uniformly normal structure, Uniformly generalized semigroup, Fixed point, Characteristic of convexity, Modulus of convexity.

\section{Introduction}

Assume that $X$ is a real Banach space with uniformly normal structure and $C$ is a nonempty closed convex subset of $X$. A mapping $T: C \rightarrow C$ is said to be a Lipschitzian mapping if, for each integer $n \geq 1$, there exist a constant $k_{n}>0$ such that

$$
\left\|T^{n} x-T^{n} y\right\| \leq k_{n}\|x-y\| \text { for all } x, y \in C .
$$

A Lipschitzian mapping is said to be a k-uniformly Lipschitzian mapping if $k_{n} \equiv k$ for all $n \geq 1$. These mappings were first studied by Goebel and Kirk [8]. They studied the existence of a fixed point of a uniformly k-Lipschitzian mapping $T$ defined on a bounded closed convex subset of a uniformly convex Banach space

\footnotetext{
${ }^{*}$ Corresponding author

Email addresses: ahsolimanm@gmail.com (Ahmed H. Soliman), barakat96@yahoo.com (Mohamed A. Barakat)
} 
$X$. They showed that such mapping $T$ has a fixed point if $k<\gamma$ where $\gamma>1$ is the unique solution of the equation

$$
\left(1-\delta_{X}(1 / \gamma)\right) \gamma=1
$$

$\delta_{X}$ being the modulus of convexity of $X$.

In 1973, Goebel and Kirk [8] then posed the question whether or not the constant $\gamma>1$ which solves the equation 1.1 is the largest number for which any k-uniformly Lipschitzian mapping $T$ with $k<\gamma$ has a fixed point.

In 1975, Lifschits [12] proved that in Hilbert space a k-uniformly Lipschitzian mapping with $k<\sqrt{2}$ has a fixed point.

Casini and Maluta [4] and Ishihara and Takahashi [10] proved that a uniformly $k$-Lipschitzian semigroup in Banach space $X$ has a common fixed point if $k<\sqrt{N(X)}$ where $N(X)$ is the uniformly normal structure coefficient.

Since then, k-uniformly Lipschitzian mapping have extensively been investigated by many authors. Moreover, some of results for uniformly Lipschitzian mapping have been extended to uniformly Lipschitzian semigroups, and even more general, to Lipschitzian semigroup; see [16, 17, 18, 19, 20, 21, 22, 23, 24, 25, 26].

Particularly, in 1993, Tan and Xu [16] answered the question of Goebel and Kirk [8] mentioned above in the negative by proving the following:

Theorem 1.1. ([16], Theorem 3.5) Let $X$ be a real uniformly convex Banach space, $C$ a nonempty closed convex subset of $X$, and $\tau=\left\{T_{s}: s \in G\right\}$ a k-uniformly Lipschitzian semigroup on $C$ with $k<\alpha$, where $\alpha>1$ is the unique solution of the equation

$$
\frac{\alpha^{2}}{N(X)} \delta_{X}^{-1}\left(1-\frac{1}{\alpha}\right)=1
$$

where $N(X)>1$ is the normal structure coefficient of $X$. Suppose there exists an $x_{0} \in C$ such that the orbit $\left\{T_{s} x_{0}: s \in G\right\}$ is bounded. then there exists $z \in C$ such that $T_{s} z=z$ for all $s \in G$.

Remark 1.2. One can prove that $\gamma<\alpha$, where $\gamma$ and $\alpha$ are the solution of equations 1.1 and 1.2 , respectively. Consequently, the constant $\gamma$ solving equation 1.1 is not the biggest number for which every k-uniformly Lipschitzian mapping $T$ with $k<\gamma$ has a fixed point. Indeed, the best possible number $\gamma$ is still unknown, even in the setting of Hilbert spaces. It is therefore an interesting question to find another constant $\alpha^{*}$ which is strictly bigger than $\alpha$ and for which every k-uniformly Lipschitzian mapping $T$ with $k<\alpha^{*}$ has a fixed point.

Some years later, Zeng and Yang [23] proved a fixed point result for Lipschitzian semigroups as follows:

Theorem 1.3. ([23], theorem 3.1) Let $C$ be a nonempty bounded subset of a uniformly convex Banach Space $X$, and let $\tau=\left\{T_{s}: s \in G\right\}$ be a k-uniformly Lipschitzian semigroup on $C$ with

$$
\liminf _{s}||\left|T_{s}\right|||<\sqrt{\gamma_{0} N(X)},
$$

where

$$
\gamma_{0}=\inf \left\{\gamma: \gamma\left(1-\delta_{X}(1 / \gamma)\right) \geq 1 / 2\right\}
$$


and $\left\|\left|T_{s} \|\right|\right.$ is the exact Lipschitzian constant of $T_{s}$. Suppose also there exists a nonempty bounded closed convex subset $E$ of $C$ with the following properties:

(P1) $x \in E$ implies $w_{w}(x) \subset E$; where $w_{w}(x)$ is the weak w-limit set of $\tau$ at $x$, i.e.,

$$
w_{w}(x)=\left\{y \in X: y=\text { weak }-\lim _{t_{\alpha}} T_{t_{\alpha}} x \text { for some subnet }\left\{t_{\alpha}\right\} \subset G\right\} .
$$

(P2) $\tau$ is asymptotically regular on $E$; i.e., $\lim _{t}\left\|T_{t+s} x-T_{t} x\right\|=0, \forall s \in G, x \in E$.

Then there exists $z \in C$ such that $T_{s} z=z$ for all $s \in G$.

Recently, Ceng, Xu and Yao [5], studied the existence of fixed points of uniformly Lipschitzian semigroups $\tau=\left\{T_{s}: s \in G\right\}$ in the setting of Banach spaces $X$ under conditions weaker than uniform convexity. More precisely, their contributions were two fold: 1.1 they replaced the uniform convexity of $X$ in Theorem 1.1 with the weaker condition of the uniformly normal structure of $X$; and 1.2 they removed the asymptotic regularity on $E$ of the semigroup $\tau=\left\{T_{s}: s \in G\right\}$ in Theorem 1.2.

In this paper, we introduce a new k-generalized Lipschitzian one parameter semigroup of self mappings and by this condition we generalize the results due to Ceng, $\mathrm{Xu}$ and Yao [5].

\section{Preliminaries}

Let $C$ be a closed convex subset of a Banach space $X$. Then the collection $\tau=\left\{T_{s}: s \in G\right\}$ of mappings of $C$ into itself is said to be generalized Lipschitzian semigroup on $C$ if the following conditions are satisfied:

(i) $T_{s t} x=T_{s} T_{t} x$ for all $s, t \in G$ and $x \in C$;

(ii) for each $x \in C$, the mapping $t \rightarrow T_{t} x$ from $G$ into $C$ is continuous;

(iii) for each $t \in G, T_{t}: X \rightarrow X$ is continuous.

(iv) for each $t \in G$, there exists a constant $k_{t}>0$ such that

$$
\left\|T_{t} x-T_{t} y\right\| \leq k_{t} \max \left\{\|x-y\|, \frac{1}{2 \omega}\left\|x-T_{t} x\right\|, \frac{1}{2 \omega}\left\|y-T_{t} y\right\|\right\} \quad \text { for all } x, y \in C,
$$

where $\omega>k, \omega>1$.

In particular, if $k_{t} \equiv k$ then $\tau=\left\{T_{s}: s \in G\right\}$ is called k-uniformly generalized Lipschitzian semigroup on $C$. Recall that $X$ is strictly convex if its unit sphere does not contain any line segments, that is, $X$ is strictly convex if and only if the following implication holds:

$$
x, y \in X, \quad\|x\|=\|y\|=1 \text { and }\|(x+y) / 2\|=1 \Rightarrow x=y .
$$

In order to measure the degree of convexity of $X$, we define its modulus of convexity $\delta_{X}:[0,2] \rightarrow[0,1]$ by

$$
\delta_{X}(\varepsilon)=\inf \{1-\|(x+y) / 2\|:\|x\| \leq 1,\|y\| \leq 1 \text { and }\|x-y\| \geq \varepsilon\} .
$$

The characteristic of convexity of $X$ is the number $\varepsilon_{0}(X)=\sup \left\{\varepsilon \in[0,2]: \delta_{X}(\varepsilon)=0\right\}$. It is easy to see [7] that $X$ is uniformly convex iff $\varepsilon_{0}(X)=0$; uniformly nonsquare iff $\varepsilon_{0}(X)<2$; and strictly convex iff $\delta(2)=1$. Moreover, if $\varepsilon_{0}(X)<1$; then $X$ has a normal structure, that is, each bounded convex subset $H$ of $X$ which contains more than one point contains a point $x_{0}$ such that $\sup \left\{\left\|x_{0}-x\right\|: x \in H\right\}<\operatorname{diam}(H)$.

The following properties of modulus $\delta_{X}$ of convexity of $X$ are quite well-known (see [9])

(a) $\delta_{X}$ is increasing on $[0,2]$, and moreover strictly increasing on $\left[\varepsilon_{0}, 2\right]$;

(b) $\delta_{X}$ is continuous on $[0,2)$ (but not necessarily at $\varepsilon=2$ );

(c) $\delta_{X}(2)=1$ iff $X$ is strictly convex;

(d) $\delta_{X}(0)=0$ and $\lim _{\varepsilon \rightarrow 2^{-}} \delta_{X}(\varepsilon)=1-\varepsilon_{0} / 2$ 
(e) $[\|a-x\| \leq r,\|a-y\| \leq r$ and $\|x-y\| \geq \varepsilon] \Rightarrow\|a-(x+y) / 2\| \leq r\left(1-\delta_{X}(\varepsilon / r)\right)$.

Recall that the normal structure coefficient $N(X)$ of $X$ is the number (see [3] )

$$
\inf \left\{\frac{\operatorname{diamK}}{r_{K}(K)}\right\}
$$

where the infimum is taken over all bounded closed convex subsets $K$ of $X$ with more than one member, and $r_{K}(K)$ and $\operatorname{diam}(K)$ are Chebyshev radii of $K$ relative to it self and the diameter of $K$, respectively, i.e., $r_{K}(K)=\inf _{x \in K} \sup _{y \in K}\|x-y\|$ and $\operatorname{diam} K=\sup _{x, y \in K}\|x-y\|$. A Banach space $X$ is said to have uniformly normal structure if $N(X)>1$. it is known that a Banach space with uniformly normal structure is reflexive and that all uniformly convex or uniformly smooth Banach spaces have uniformly normal structure (see, e.g., [26]). It is also been computed that $N(H)=\sqrt{2}$ for a Hilbert spaces $H$. The computations of the normal structure coefficient $N(X)$ for general Banach spaces look however complicated. No exact values of $N(X)$ are known except for some special cases (e.g., Hilbert and $L^{p}$ spaces). In general, we have the following lower bounded for $N(X)$ (see [3, 14, 1])

$$
N(X) \geq \frac{1}{1-\delta_{X}(1)}
$$

Other lower bounds for $N(X)$ in terms of some Banach space parameters or constants can be found in [11, 15].

Tan and $\mathrm{Xu}$ [16] have also proven that if $X$ is uniformly convex and $\gamma>1$ is the unique solution of the equation 1.1, then $N(X)>\gamma$. Note that for a Hilbert space $H$, we have $N(H)=\sqrt{2}$ and $\gamma=\sqrt{5} / 2$. Suppose $X$ is uniformly convex Banach space. Then it is easily seen that the equation

$$
\alpha^{2} \delta_{X}^{-1}\left(1-\frac{1}{\alpha}\right) \tilde{N}(X)=1
$$

has a unique solution $\alpha>1$, where $\tilde{N}(X)=1 / N(X)$. Tan and Xu [16] proved that if $\gamma>1$ and $\alpha>1$ are the solution of 1.1 and 2.1 respectively, then $\gamma<\alpha$. Note that $\gamma=\sqrt{5} / 2$, and $\alpha=\frac{1}{\sqrt{\sqrt{3}-1}}>\gamma$.

We need the notation of asymptotic centers, due to Edelstein [6]. Let $C$ be a nonempty closed convex subset of a Banach space $X$ and let $\left\{x_{t}: t \in G\right\}$ be a bounded net of elements of $X$. Then the asymptotic radius and asymptotic center of $\left\{x_{t}\right\}_{t \in G}$ with respect to $C$ are the number

$$
r_{C}\left\{x_{t}\right\}=\inf _{y \in C} \limsup _{t}\left\|x_{t}-y\right\|,
$$

and respectively, the (possibly empty) set

$$
A_{C}\left(\left\{x_{t}\right\}\right)=\left\{y \in C: \limsup _{t}\left\|x_{t}-y\right\|=r_{C}\left(\left\{x_{t}\right\}\right)\right\} .
$$

Lemma 2.1. ([16], Lemma 2.1) If $C$ is a nonempty closed convex subset of a reflexive Banach space $X$, then for every bounded net $\left\{x_{t}\right\}_{t \in G}$ of elements of $X, A_{C}\left(\left\{x_{t}\right\}\right)$ is a nonempty bounded closed convex subset of $C$. In particular, if $X$ is a uniformly convex Banach space, then $A_{C}\left(\left\{x_{t}\right\}\right)$ consists of a single point.

The following lemma can be proven in exactly the same way as in Lim [13] for sequences and the proof is thus omitted here.

Lemma 2.2. ([16], lemma 2.2) Suppose $X$ is a Banach space with uniformly normal structure. Then for every bounded net $\left\{x_{t}\right\}_{t \in G}$ of elements of $X$ there exists $y \in \overline{c o}\left(\left\{x_{t}: t \in G\right\}\right)$ such that

$$
\limsup _{t}\left\|x_{t}-y\right\| \leq \widetilde{N}(X) D\left(\left\{x_{t}\right\}\right)
$$

where $\tilde{N}(X)=1 / N(X)$, and $\overline{c o}(E)$ is the closure of the convex hull of a set $E \subset X$ and $D\left(\left\{x_{t}\right\}\right)=$ $\lim _{t}\left(\sup \left\{\left\|x_{i}-x_{j}\right\|: t \leq i, j \in G\right\}\right)$ is the asymptotic diameter of $\left\{x_{t}\right\}$. 


\section{Main results}

The following lemma plays an important role in proving our results.

Lemma 3.1. Let $\left\{T_{s} x_{0} ; s \in G\right\}$ be a bounded for some $x_{0} \in C$ and $\tau=\left\{T_{s} ; s \in G\right\}$ k-uniformly generalized Lipschitzian semigroup on $C$, then $\left\{T_{s} x ; s \in G\right\}$ is bounded for each $x \in C$.

We next present the first result of this paper which weakens the uniform convexity assumption in theorem 1.1.

Theorem 3.2. Suppose $X$ is a real Banach space with $N(X)>\max \left(1, \varepsilon_{0}\right), C$ is a nonempty closed convex subset of $X$, and $\tau=\left\{T_{s}: s \in G\right\}$ is a k-uniformly generalized Lipschitzian semigroup on $C$ which satisfy the condition (iv) with $\omega<\alpha_{*}$. Here $\varepsilon_{0}$ is the characteristic of convexity of $X$ and

$$
\alpha_{*}=\sup \left\{\alpha: \alpha^{2} \delta_{X}^{-1}\left(1-\frac{1}{\alpha}\right) N(X)^{-1} \leq 1 \text { and } 1-\frac{1}{\alpha} \in\left(0,1-\frac{1}{2} \varepsilon_{0}\right)\right\} .
$$

If $\left\{T_{s} x_{0}: s \in G\right\}$ is bounded for some $x_{0} \in C$, then there exists $z \in C$ such that $T_{s} z=z$ for all $s \in G$.

Proof. Put $\widetilde{N}(X)=N(X)^{-1}$. Observe that the set

$$
\left\{\alpha: \alpha^{2} \delta_{X}^{-1}\left(1-\frac{1}{\alpha}\right) N(X)^{-1} \leq 1 \text { and } 1-\frac{1}{\alpha} \in\left(0,1-\frac{1}{2} \varepsilon_{0}\right)\right\} \neq \phi .
$$

Indeed, by properties (a),(b),(d) of the modulus $\delta_{x}$ of convexity of $X$, we see that the mapping

$$
\delta_{x}:\left[\varepsilon_{0}, 2\right) \rightarrow \delta_{x}\left(\left[\varepsilon_{0}, 2\right)\right)=\left[0,1-\frac{1}{2} \varepsilon_{0}\right)
$$

is strictly increasing and continuous, and hence a bijection. Thus, we deduce that

$$
\lim _{\alpha \rightarrow 1^{+}} \alpha^{2} \delta_{X}^{-1}\left(1-\frac{1}{\alpha}\right) N(X)^{-1}=\delta_{X}^{-1}(0) \tilde{N}(X)=\varepsilon_{0} \tilde{N}(X)<1 .
$$

which implies that there exists $\alpha_{0}>1$ such that $\alpha_{0}^{2} \delta_{X}^{-1}\left(1-\frac{1}{\alpha_{0}}\right) \widetilde{N}(X)<1$ and

$$
1-\frac{1}{\alpha_{0}} \in \delta_{x}\left(\left[\varepsilon_{0}, 2\right)\right)=\left[0,1-\frac{1}{2} \varepsilon_{0}\right) .
$$

This verifies our assertion 3.2 .

Since $X$ has a uniformly normal structure, $X$ is reflexive. Due to the boundedness of $\left\{T_{s} x_{0}: s \in G\right\}$ and by Lemma 2.1, we get that $A_{C}\left(\left\{T_{t} x_{0}\right\}_{t \in G}\right)$ is nonempty bounded closed convex subset of $C$. Then we can choose $x_{1} \in A_{C}\left(\left\{T_{t} x_{0}\right\}_{t \in G}\right)$ such that

$$
\limsup _{t}\left\|T_{t} x_{0}-x_{1}\right\|=\inf _{y \in C} \limsup _{t}\left\|T_{t} x_{0}-y\right\| .
$$

Since $\tau$ is $k$-uniformly generalized Lipschitzian property, by lemma 3.1, we know that $T_{t} x_{1}$ remains bounded. Consequently we can choose $x_{2} \in A_{C}\left(\left\{T_{t} x_{1}\right\}_{t \in G}\right)$ such that

$$
\limsup _{t}\left\|T_{t} x_{1}-x_{2}\right\|=\inf _{y \in C} \limsup _{t}\left\|T_{t} x_{1}-y\right\| .
$$

Continuing this process, we can construct a sequence $\left\{x_{n}\right\}_{n=0}^{\infty}$ in $C$ with the properties:

(i) for each $n \geq 0,\left\{T_{t} x_{n}\right\}_{t \in G}$ is bounded;

(ii) for each $n \geq 0, x_{n+1} \in A_{C}\left(\left\{T_{t} x_{n}\right\}_{t \in G}\right)$; that is $x_{n+1}$ is a point in $C$ such that

$$
\lim _{t}\left\|T_{t} x_{n}-x_{n+1}\right\|=\inf _{y \in C} \lim _{t}\left\|T_{t} x_{n}-y\right\| .
$$


Write $r_{n}=r_{C}\left(\left\{T_{t} x_{n}\right\}_{t \in G}\right)$. Then by Lemma 2.2 we have

$$
\begin{gathered}
r_{n}=\limsup _{t}\left\|T_{t} x_{n}-x_{n+1}\right\| \leq \widetilde{N}(X) D\left(\left\{T_{t} x_{n}\right\}_{t \in G}\right)=\widetilde{N}(X) \lim _{t}\left(\sup \left\{\left\|T_{i} x_{n}-T_{j} x_{n}\right\|: t \leq i, j \in G\right\}\right) \\
\leq \widetilde{N}(X) k \lim _{t}\left(\sup \max \left\{\left\|x_{n}-T_{j-i} x_{n}\right\|, \frac{1}{2 \omega}\left\|x_{n}-T_{i} x_{n}\right\|, \frac{1}{2 \omega}\left\|T_{j} x_{n}-T_{j-i} x_{n}\right\|\right\}\right. \\
\leq \widetilde{N}(X) k \lim _{t}\left(\sup \max \left\{d\left(x_{n}\right), \frac{1}{2 \omega} d\left(x_{n}\right), \frac{1}{\omega} d\left(x_{n}\right)\right\} \leq \widetilde{N}(X) \cdot k \cdot d\left(x_{n}\right),\right.
\end{gathered}
$$

that is,

$$
r_{n} \leq \tilde{N}(X) \cdot k \cdot d\left(x_{n}\right) \leq \tilde{N}(X) \cdot \omega \cdot d\left(x_{n}\right)
$$

Where

$$
d\left(x_{n}\right)=\sup \left\{\left\|x_{n}-T_{t} x_{n}\right\|: t \in G\right\} .
$$

We may assume that $d\left(x_{n}\right)>0$ for all $n \geq 0$ (since otherwise $x_{n}$ is a common fixed point of the semigroup $\tau$ and the proof is finished). Let $n \geq 0$ be fixed and let $\varepsilon>0$ be small enough. We can choose $j \in G$ such that

$$
\left\|T_{j} x_{n+1}-x_{n+1}\right\|>d\left(x_{n+1}\right)-\varepsilon
$$

and then choose $s_{0} \in G$ so large that

$$
\left\|T_{s} x_{n}-x_{n+1}\right\|<r_{n}+\varepsilon<\omega\left(r_{n}+\varepsilon\right)
$$

for all $s \geq s_{0}$. It turns out, for $s \geq s_{0}+j$,

$$
\begin{gathered}
\left\|T_{s} x_{n}-T_{j} x_{n+1}\right\| \leq k \max \left\{\left\|T_{s-j} x_{n}-x_{n+1}\right\|, \frac{1}{2 \omega}\left\|T_{s} x_{n}-T_{s-j} x_{n}\right\|, \frac{1}{2 \omega}\left\|x_{n+1}-T_{j} x_{n+1}\right\|\right\} \\
\leq k \max \left\{r_{n}+\varepsilon, \frac{1}{\omega}\left(r_{n}+\varepsilon\right), \frac{1}{2 \omega}\left(r_{n}+\varepsilon\right)+\frac{1}{2 \omega}\left\|T_{s} x_{n}-T_{j} x_{n+1}\right\|\right\} .
\end{gathered}
$$

If

$$
\max \left\{r_{n}+\varepsilon, \frac{1}{\omega}\left(r_{n}+\varepsilon\right), \frac{1}{2 \omega}\left(r_{n}+\varepsilon\right)+\frac{1}{2 \omega}\left\|T_{s} x_{n}-T_{j} x_{n+1}\right\|\right\}=\frac{1}{2 \omega}\left(r_{n}+\varepsilon\right)+\frac{1}{2 \omega}\left\|T_{s} x_{n}-T_{j} x_{n+1}\right\|,
$$

then we have:

$$
\left\|T_{s} x_{n}-T_{j} x_{n+1}\right\| \leq \frac{k}{2 \omega}\left(r_{n}+\varepsilon\right)+\frac{k}{2 \omega}\left\|T_{s} x_{n}-T_{j} x_{n+1}\right\| \leq \frac{1}{2}\left(r_{n}+\varepsilon\right)+\frac{1}{2}\left\|T_{s} x_{n}-T_{j} x_{n+1}\right\|,
$$

hence

$$
\left\|T_{s} x_{n}-T_{j} x_{n+1}\right\| \leq r_{n}+\varepsilon<\omega\left(r_{n}+\varepsilon\right) .
$$

Then it follows from property (e) that

$$
\left\|T_{s} x_{n}-\frac{1}{2}\left(x_{n+1}+T_{j} x_{n+1}\right)\right\| \leq \omega\left(r_{n}+\varepsilon\right)\left(1-\delta_{X}\left(\frac{d\left(x_{n+1}\right)-\varepsilon}{\omega\left(r_{n}+\varepsilon\right)}\right)\right)
$$

for $s \geq s_{0}+j$ and hence

$$
r_{n} \leq \limsup _{s}\left\|T_{s} x_{n}-\frac{1}{2}\left(x_{n+1}+T_{j} x_{n+1}\right)\right\| \leq \omega\left(r_{n}+\varepsilon\right)\left(1-\delta_{X}\left(\frac{d\left(x_{n+1}\right)-\varepsilon}{\omega\left(r_{n}+\varepsilon\right)}\right)\right) .
$$

Taking the limit as $\varepsilon \rightarrow 0$ we obtain

$$
r_{n} \leq \omega r_{n}\left(1-\delta_{X}\left(\frac{d\left(x_{n+1}\right)}{\omega r_{n}}\right)\right) .
$$


This implies that

$$
\begin{gathered}
\delta_{X}\left(\frac{d\left(x_{n+1}\right)}{\omega r_{n}}\right) \leq 1-\frac{1}{\omega} \\
d\left(x_{n+1}\right) \leq \omega r_{n} \delta_{X}^{-1}\left(1-\frac{1}{\omega}\right)
\end{gathered}
$$

Indeed, if $d\left(x_{n+1}\right) /\left(\omega r_{n}\right) \in\left[0, \varepsilon_{0}\right)$, then noticing that $\delta_{X}:\left[\varepsilon_{0}, 2\right) \rightarrow\left[0,1-\varepsilon_{0} / 2\right)$ is a bijection and that $1-\frac{1}{\omega}$ lies in $\left[0,1-\varepsilon_{0} / 2\right)$ by assumption $k<\omega<\alpha_{*}$, we have $\delta_{X}^{-1}\left(1-\frac{1}{\omega}\right) \geq \varepsilon_{0}$; hence $d\left(x_{n+1}\right) /\left(\omega r_{n}\right) \leq \delta_{X}^{-1}\left(1-\frac{1}{\omega}\right)$ and 3.5 follows. If $d\left(x_{n+1}\right) /\left(\omega r_{n}\right) \in\left[\varepsilon_{0}, 2\right]$, then it is clear that $d\left(x_{n+1}\right) /\left(\omega r_{n}\right) \leq \delta_{X}^{-1}\left(1-\frac{1}{\omega}\right)$. This also shows that 3.6 is true.

Therefore, utilizing 3.3 and 3.5 , we obtain

$$
d\left(x_{n+1}\right) \leq \omega^{2} \widetilde{N}(X) \delta_{X}^{-1}\left(1-\frac{1}{\omega}\right) d\left(x_{n}\right) .
$$

Write $A=\omega^{2} \widetilde{N}(X) \delta_{X}^{-1}\left(1-\frac{1}{\omega}\right)$. Then $A<1$. Indeed, from the assumption that $\omega<\alpha_{*}$ it follows that there exists an $\tilde{\alpha}>\omega$ such that

$$
\tilde{\alpha}^{2} \widetilde{N}(X) \delta_{X}^{-1}\left(1-\frac{1}{\tilde{\alpha}}\right) \leq 1 \text { and }\left(1-\frac{1}{\tilde{\alpha}}\right) \in \delta_{X}\left(\left(\varepsilon_{0}, 2\right)\right) .
$$

It then turns out that $\delta_{X}^{-1}\left(1-\frac{1}{\omega}\right)<\delta_{X}^{-1}\left(1-\frac{1}{\tilde{\alpha}}\right)$, and

$$
A=\omega^{2} \widetilde{N}(X) \delta_{X}^{-1}\left(1-\frac{1}{\omega}\right)<\tilde{\alpha}^{2} \widetilde{N}(X) \delta_{X}^{-1}\left(1-\frac{1}{\tilde{\alpha}}\right) \leq 1 .
$$

Hence, it is follows from 3.6 that

$$
d\left(x_{n}\right) \leq A d\left(x_{n-1}\right) \leq \ldots \leq A^{n} d\left(x_{0}\right) .
$$

Since

$$
\left\|x_{n+1}-x_{n}\right\| \leq \limsup _{t}\left\|T_{t} x_{n}-x_{n+1}\right\|+\limsup _{t}\left\|T_{t} x_{n}-x_{n}\right\| \leq r_{n}+d\left(x_{n}\right) \leq 2 d\left(x_{n}\right) .
$$

We get from 3.7 that $\sum_{n=1}^{\infty}\left\|x_{n+1}-x_{n}\right\|<\infty$, and hence $\left\{x_{n}\right\}$ is a norm-Cauchy. Let $z=\|\|-.\lim _{n} x_{n}$. Finally, we have for each $s \in G$,

$$
\begin{gathered}
\left\|z-T_{s} z\right\| \leq\left\|z-x_{n}\right\|+\left\|T_{s} x_{n}-x_{n}\right\|+\left\|T_{s} x_{n}-T_{s} z\right\| \leq\left\|z-x_{n}\right\|+d\left(x_{n}\right)+k \max \left\{\left\|z-x_{n}\right\|, \frac{1}{2 \omega} d\left(x_{n}\right), \frac{1}{2 \omega}\left\|z-T_{s} z\right\|\right\} \\
\leq\left\|z-x_{n}\right\|+d\left(x_{n}\right)+k\left\|z-x_{n}\right\|+\frac{k}{2 \omega} d\left(x_{n}\right)+\frac{k}{2 \omega}\left\|z-T_{s} z\right\|<\left\|z-x_{n}\right\|+d\left(x_{n}\right)+k\left\|z-x_{n}\right\|+\frac{k}{2 \omega} d\left(x_{n}\right)+\frac{1}{2}\left\|z-T_{s} z\right\| \\
\left\|z-T_{s} z\right\| \leq 2(k+1)\left\|z-x_{n}\right\|+(k+2) d\left(x_{n}\right) \rightarrow 0 \text { as } n \rightarrow \infty
\end{gathered}
$$

Hence, $T_{s} z=z$ for all $s \in G$ and the proof is complete.

Theorem 3.3. Let $C$ be a nonempty bounded subset of a uniformly convex Banach space $X$, and $\tau=\left\{T_{s}\right.$ : $s \in G\}$ be a k-uniformly generalized Lipschitzian semigroup on $C$ which satisfy the condition (iv) with

$$
\omega<\sqrt{\gamma_{0} N(X)}, \text { where } \gamma_{0}=\inf \left\{\gamma \geq 1: \gamma\left(1-\delta_{X}(1 / \gamma)\right) \geq 1 / 2 .\right.
$$

Suppose also there exists a nonempty bounded closed convex subset $E$ of $C$ with the following property $(\Re)$ : $(\Re) x \in E$ implies $w_{w}(x) \subset E$.

Then there exists $z \in E$ such that $T_{s} z=z$ for all $s \in G$. 
Proof. Take an $x_{0} \in E$ and, consider for each $t \in G$, the bounded net $\left\{T_{s} x_{0}: t \leq s \in G\right\}$.

According to Lemma 2.2, we have a $y_{t} \in \overline{c o}\left\{T_{s} x_{0}: t \leq s \in G\right\}$ such that

$$
\limsup _{s}\left\|T_{s} x_{0}-y_{t}\right\| \leq \tilde{N}(X) D\left(\left\{T_{s} x_{0}\right\}_{t \leq s \in G}\right)
$$

where $\widetilde{N}(X)=1 / N(X)$ and $D\left(\left\{T_{s} x_{0}\right\}_{t \leq s \in G}\right)$ denotes the asymptotic diameter of the net $\left\{z_{l}\right\}$ i.e, the number

$$
\lim _{t}\left(\sup \left\{\left\|z_{i}-z_{j}\right\|: t \leq i, j \in G\right\}\right)
$$

Since $X$ is reflexive, $\left\{y_{t}\right\}$ admits a subnet $\left\{y_{t_{\beta}}\right\}$ converging weakly to some $x_{1} \in X$. From 3.9 and the weakly lower semicontinuity of the functional $\lim \sup _{t}\left\|T_{t} x_{0}-y\right\|$, it follows that

$$
\underset{t}{\limsup }\left\|T_{t} x_{0}-x_{1}\right\| \leq \widetilde{N}(X) D\left(\left\{T_{t} x_{0}\right\}_{t \in G}\right) .
$$

It is also seen that $x_{1} \in \bigcap_{t \in G} \overline{c o}\left\{T_{s} x_{0}: t \leq s \in G\right\}$ and that

$$
\left\|z-x_{1}\right\| \leq \limsup _{t}\left\|z-T_{t} x_{0}\right\| \text { for all } z \in X .
$$

Observing Property $(\Re)$ and the fact that $\bigcap_{t \in G} \overline{c o}\left\{T_{s} x_{0}: t \leq s \in G\right\}=\overline{c o}\left\{w_{w}\left(x_{0}\right)\right\}$ which is easy to prove by using the Separation Theorem(see[2]), we know that $x_{1}$ actually lies in $E$. So we can repeat the above process and obtain a sequence $\left\{x_{n}\right\}_{n=0}^{\infty}$ in $E$ with the properties: for all nonnegative integers $n \geq 0$,

$$
\limsup _{t}\left\|T_{t} x_{n}-x_{n+1}\right\| \leq \widetilde{N}(X) D\left(\left\{T_{t} x_{n}\right\}_{t \in G}\right) .
$$

and

$$
\left\|z-x_{n+1}\right\| \leq \limsup _{t}\left\|z-T_{t} x_{n}\right\| \text { for all } z \in X
$$

Write $r_{n}=\lim \sup _{t}\left\|T_{t} x_{n}-x_{n+1}\right\|$ and $d\left(x_{n}\right)=\sup \left\{\left\|x_{n}-T_{t} x_{n}\right\|: t \in G\right\}$. This in view of 3.12, we have

$$
\begin{gathered}
r_{n}=\limsup _{t}\left\|T_{t} x_{n}-x_{n+1}\right\| \leq \tilde{N}(X) D\left(\left\{T_{t} x_{n}\right\}_{t \in G}\right)=\widetilde{N}(X) \lim _{t}\left(\sup \left\{\left\|T_{i} x_{n}-T_{j} x_{n}\right\|: t \leq i, j \in G\right\}\right) \\
\leq \tilde{N}(X) k \lim _{t}\left(\sup \max \left\{\left\|x_{n}-T_{j-i} x_{n}\right\|, \frac{1}{2 \omega}\left\|x_{n}-T_{i} x_{n}\right\|, \frac{1}{2 \omega}\left\|T_{j} x_{n}-T_{j-i} x_{n}\right\|\right\}\right. \\
\leq \tilde{N}(X) k \lim _{t}\left(\max \left\{d\left(x_{n}\right), \frac{1}{2 \omega} d\left(x_{n}\right), \frac{1}{\omega} d\left(x_{n}\right)\right\}\right) \leq \tilde{N}(X) \cdot k \cdot d\left(x_{n}\right)<\tilde{N}(X) \cdot \omega \cdot d\left(x_{n}\right),
\end{gathered}
$$

that is,

$$
r_{n}<\tilde{N}(X) \cdot \omega \cdot d\left(x_{n}\right)
$$

We may assume that $d\left(x_{n}\right)>0$ for all $n \geq 0$. Let $n \geq 0$ be fixed and let $\varepsilon>0$ be small enough. First choose $j \in G$ such that,

$$
\left\|T_{j} x_{n+1}-x_{n+1}\right\|>d\left(x_{n+1}\right)-\varepsilon
$$

and then choose $s_{0} \in G$ so large that

$$
\left\|T_{s} x_{n}-x_{n+1}\right\|<r_{n}+\varepsilon<\omega\left(r_{n}+\varepsilon\right)
$$

for all $s \geq s_{0}$. Then we have, for $s \geq s_{0}+j$,

$$
\left\|T_{s} x_{n}-T_{j} x_{n+1}\right\| \leq k \max \left\{\left\|T_{s-j} x_{n}-x_{n+1}\right\|, \frac{1}{2 \omega}\left\|T_{s} x_{n}-T_{s-j} x_{n}\right\|, \frac{1}{2 \omega}\left\|x_{n+1}-T_{j} x_{n+1}\right\|\right\}
$$




$$
\leq k \max \left\{r_{n}+\varepsilon, \frac{1}{\omega}\left(r_{n}+\varepsilon\right), \frac{1}{2 \omega}\left(r_{n}+\varepsilon\right)+\frac{1}{2 \omega}\left\|T_{s} x_{n}-T_{j} x_{n+1}\right\|\right\} .
$$

If

$$
\max \left\{r_{n}+\varepsilon, \frac{1}{\omega}\left(r_{n}+\varepsilon\right), \frac{1}{2 \omega}\left(r_{n}+\varepsilon\right)+\frac{1}{2 \omega}\left\|T_{s} x_{n}-T_{j} x_{n+1}\right\|\right\}=\frac{1}{2 \omega}\left(r_{n}+\varepsilon\right)+\frac{1}{2 \omega}\left\|T_{s} x_{n}-T_{j} x_{n+1}\right\|,
$$

then we have

$$
\left\|T_{s} x_{n}-T_{j} x_{n+1}\right\| \leq\left(r_{n}+\varepsilon\right)<\omega\left(r_{n}+\varepsilon\right)
$$

Then it follows from property (e) that, for $s \geq s_{0}+j$,

$$
\left\|T_{s} x_{n}-\frac{1}{2}\left(x_{n+1}+T_{j} x_{n+1}\right)\right\| \leq \omega\left(r_{n}+\varepsilon\right)\left(1-\delta_{X}\left(\frac{d\left(x_{n+1}\right)-\varepsilon}{\omega\left(r_{n}+\varepsilon\right)}\right)\right) .
$$

Hence from 3.13 (taking $\left.z:=\left(x_{n+1}+T_{j} x_{n+1}\right) / 2\right)$ we obtain

$$
\begin{aligned}
\frac{1}{2}\left(d\left(x_{n+1}\right)-\varepsilon\right)< & \left\|\frac{1}{2}\left(T_{j} x_{n+1}-x_{n+1}\right)\right\| \leq\left\|T_{j} x_{n+1}-\frac{1}{2}\left(x_{n+1}+T_{j} x_{n+1}\right)\right\| \\
& \leq \limsup _{t}\left\|T_{t} x_{n}-\frac{1}{2}\left(x_{n+1}+T_{j} x_{n+1}\right)\right\| \\
& \leq \omega\left(r_{n}+\varepsilon\right)\left(1-\delta_{X}\left(\frac{d\left(x_{n+1}\right)-\varepsilon}{\omega\left(r_{n}+\varepsilon\right)}\right)\right) .
\end{aligned}
$$

Taking the limit as $\varepsilon \rightarrow 0$ we have

$$
\frac{1}{2} d\left(x_{n+1}\right) \leq \omega r_{n}\left(1-\delta_{X}\left(\frac{d\left(x_{n+1}\right)}{\omega r_{n}}\right)\right) .
$$

On other hand, we easily find by 3.13 that, for each $j \in G$,

$$
\left.\| T_{j} x_{n+1}-x_{n+1}\right)\left\|\leq \limsup _{t}\right\| T_{j} x_{n+1}-T_{t} x_{n} \|<\omega r_{n} .
$$

It turns out that

$$
d\left(x_{n+1}\right)<\omega r_{n}
$$

Combining 3.16 and 3.17 and using the definition of $\gamma_{0}$ in 3.8 , we infer that $\left(\omega r_{n}\right) / d\left(x_{n+1}\right) \geq \gamma_{0}$. It turns out from 3.14 that

Consequently, we obtain

$$
d\left(x_{n+1}\right) \leq \frac{\omega}{\gamma_{0}} r_{n}<\frac{\omega^{2}}{\gamma_{0} N(X)} d\left(x_{n}\right) .
$$

$$
d\left(x_{n}\right) \leq A d\left(x_{n-1}\right) \leq \ldots \leq A^{n} d\left(x_{0}\right),
$$

where $A=\omega^{2}\left[\gamma_{0} N(X)\right]^{-1}<1$ by assumption. Noticing that

$$
\begin{aligned}
& \left\|x_{n+1}-x_{n}\right\| \leq \limsup _{t}\left\|T_{t} x_{n}-x_{n+1}\right\|+\limsup _{t}\left\|T_{t} x_{n}-x_{n}\right\| \\
& \leq r_{n}+d\left(x_{n}\right) \leq(1+k \widetilde{N}(X)) d\left(x_{n}\right) \leq(1+k \widetilde{N}(X)) A^{n} d\left(x_{0}\right),
\end{aligned}
$$

that $\sum_{n=1}^{\infty}\left\|x_{n+1}-x_{n}\right\|$ is convergent. This implies that $\left\{x_{n}\right\}$ is strongly convergent. Let $z=\|\|-.\lim _{n} x_{n}$. Then, we have for each $s \in G$,

$$
\begin{gathered}
\left\|z-T_{s} z\right\| \leq\left\|z-x_{n}\right\|+\left\|T_{s} x_{n}-x_{n}\right\|+\left\|T_{s} x_{n}-T_{s} z\right\| \leq\left\|z-x_{n}\right\|+d\left(x_{n}\right)+k \max \left\{\left\|z-x_{n}\right\|, \frac{1}{2 \omega} d\left(x_{n}\right), \frac{1}{2 \omega}\left\|z-T_{s} z\right\|\right\} \\
\leq\left\|z-x_{n}\right\|+d\left(x_{n}\right)+k\left\|z-x_{n}\right\|+\frac{k}{2 \omega} d\left(x_{n}\right)+\frac{k}{2 \omega}\left\|z-T_{s} z\right\|<\left\|z-x_{n}\right\|+d\left(x_{n}\right)+k\left\|z-x_{n}\right\|+\frac{k}{2 \omega} d\left(x_{n}\right)+\frac{1}{2 \omega}\left\|z-T_{s} z\right\| \\
\left\|z-T_{s} z\right\| \leq 2(k+1)\left\|z-x_{n}\right\|+(k+2) d\left(x_{n}\right) \rightarrow 0 \text { as } n \rightarrow \infty
\end{gathered}
$$

Hence, $T_{s} z=z$ for all $s \in G$ and the proof is complete. 


\section{Acknowledgements:}

Both the authors are grateful to an anonymous referee for his fruitful comments.

\section{References}

[1] A. G. Aksoy and M. A. Khamsi, Nonstandard methods in fixed point theory, Springer, New York, (1990). 2

[2] R. E. Bruck, On the almost-convergence of iterates of a nonexpansive mappings in Hilbert space and the structure of the weak-limit set, Israel J. Math. 29 (1978), 1-16. 3

[3] W. L. Bynum, Normal structure coefficients for Banach spaces, Pacific J. Math. 86 (1980), 427-436. 2

[4] E. Casini and E. Maluta, Fixed points of uniformly Lipschitzian mappings in spaces with uniformly normal structure, Nonlinear Anal. 9 (1985), 103-108. 1

[5] L.C. Ceng, H. K. Xu and J.C. Yao, Uniformly normal structure and uniformly Lipschitzian semigroups, Nonlinear Anal. (2010), doi:10.1016/j.na. 2010.07. 044. (document), 1

[6] M. Edelstein, The construction of an asymptotic center with a fixed point property, Bull. Amer. Math. Soc. 78 (1972), 206-208. 2

[7] K. Goebel, Convexity of balls and fixed point theorems for mappings with nonexpansive square, Compos. Math. 22 (1970), 269-274. 2

[8] K. Goebel and W. A. Kirk, A fixed point theorem for transformations whose iterates have uniform Lipschitz constant, Studia Math. 47 (1973), 135-140. 1,1

[9] K. Goebel and S. Reich, Uniform convexity, hyperbolic geometry and nonexpansive mappings, in: pure and Applied Math., A series of Monoghraph and Textbooks, 83, Marcel Dekker, New York, (1984). 2

[10] H. Ishihara and W. Takahashi, Fixed point theorems for unformly Lipschitzian semigroups in Hilbert spaces, J. Math. Anal. Appl. 127 (1987), 206-210. 1

[11] M. Kato, L. Maligranda, and Y. Takahashi, On James and Jordan-von Neumann constants and normal structure coefficient in Banach spaces, Studia Math. 144 (2001), no. 3, 275-293. 2

[12] E. A. Lifschits, Fixed point theorems for operators in strongly convex spaces, Voronez Gos. Univ. Trudy Math. Fak. 16 (1975), 23-28. 1

[13] T. C. Lim, On the normal structure coefficient and the bounded sequence coefficient, Proc. Amer. Math. Soc. 88 (1983) 262-264. 2

[14] E. Maluta, Uniform normal structure and related coefficients, Pacific J. Math. 111 (1984), 357-369. 2

[15] S. Prus and M. Szczepanik, New coefficients related to uniform normal structure, Nonlinear and Convex Anal. 2 (2001), no. 3, 203-215. 2

[16] K. K. Tan and H. K. Xu, Fixed point theorems for Lipschitzian semigroups in Banach spaces, Nonlinear Anal. 20 (1993), 395-404. 1, 1.1, 2, 2, 2.1, 2.2

[17] X. Wu J. C. Yao and L. C. Zeng, Uniformly normal structure and strong convergences theorems for asymptoticlly pseudocontractive mapping, J. Nonlinear convex Anal. 6 (3) (2005), 453-463. 1

[18] H. K. Xu, Fixed point theorems for uniformly Lipschitzian semigroups in uniformly convex spaces, J. Math. Anal. Appl. 152 (1990), 391-398. 1

[19] J. C. Yao and L. C. Zeng, A fixed point theorem for asymptotically regular semigroups in metric spaces with uniform normal structure, J. Nonlinear convex Anal. 8 (1) (2007), 153-163. 1

[20] L. C. Zeng, Fixed point theorems for nonlinear semigroups of Lipschitzian mappings in uniformly convex spaces, Chinese Quart. J. Math. 9 (4) (1994), 64-73. 1

[21] L. C. Zeng, On the existence of fixed points and nonlinear ergodic retractions for Lipschitzian semigroups without convexity, Nonlinear Anal. 24 (1995), 1347-1359. 1

[22] L. C. Zeng, Fixed point theorems for asymptotically regular Lipschitzian semigroups in Banach spaces, Chinese Ann. Math. 16A (6) (1995), 744-751. 1

[23] L. C. Zeng Y. L. Yang, On the existence of fixed points for Lipschitzian semigroups in Banach spaces, Chinese Ann. Math. 22B (3) (2001), 397-404. 1, $1,1.3$

[24] L. C. Zeng, Fixed point theorems for asymptotically regular semigroups in Banach spaces, Chinese Ann. Math. 23A (6) (2002), 699-706. 1 ]

[25] L. C. Zeng, Weak uniform normal structure and fixed points of asymptotically regular semigroups, Acta. Math. Sin. (English Series) 20 (6) (2004), 977-982. 1

[26] L. C. Zeng, Uniform normal structure and solutions of Reich's open question, Appl. Math. Mech. 26 (9) (2005), 1204-1211. 